95\%IC 1.307-16.744) (figure 1B). The time-dependent model including SSM values at baseline and at 6 months after EOT predicted post-SVR HD development better than the models including LSM and its changes after therapy.

Conclusions The risk of $\mathrm{HD}$ is markedly reduced after DAA therapy, but not abolished. SSM is confirmed as an accurate surrogate of portal hypertension, able to stratify for the risk of HD development after DAA therapy more accurately than LSM.

\section{IDDF2019-ABS-0196 LONG TERM OUTCOMES OF UTILIZING DONATION AFTER CIRCULATORY DEATH GRAFTS IN LIVER TRANSPLANTATION - AN AUSTRALIAN 12-YEAR COHORT STUDY}

Vinay Sastry*, Keval Pandya, Mara Panlilio, Claire West, Susan Virtue, Mark Wells, Michael Crawford, Carlo Pulitano, Simone Strasser, Geoff McCaughan, Avik Majumdar, Ken Liu. Australian National Liver Transplant Unit, Royal Prince Alfred Hospital, Sydney, Australia

\subsection{6/gutjnl-2019-IDDFabstracts. 14}

Background Organ shortage for liver transplantation (LT) is a major problem worldwide. Use of donation circulatory death donors (DCD) has been one strategy to expand the donor pool; however, data on long term outcomes in DCD graft recipients are mixed. We studied the characteristics, utilization and recipient outcomes of DCDs.

Methods We retrospectively studied adults who underwent deceased LT between 2006-2018. Donor and recipient data at LT and recipient outcomes were collected from a prospective database. Criteria for DCD selection at our center include all of the following: donor age $<50$ years, BMI $<30 \mathrm{~kg} / \mathrm{m}^{2}$, and circulatory arrest within 30 minutes of treatment withdrawal in otherwise suitable liver donors. The primary outcome of interest was graft survival (time to retransplantation or death).

Results During the study period (median follow-up 50.6months), 739 donors were utilized for LT with $53(7.2 \%)$ being DCDs. Compared to donation after brain death donors (DBDs), DCDs were younger (30 vs. 50years), more likely to have history of predonation cardiac arrest $(71.2 \%$ vs. $34.8 \%)$, had longer intubation time (3 vs. 2days), less inotrope requirements $(32.7 \%$ vs. $7.8 \%$ on no agents) and higher AST (59 vs. 46U/L) (median values presented, all $P<0.01$ ). DCDs had shorter cold ischemia time $(5.75$ vs. 6.85 hours, $P=0.005)$ and

A

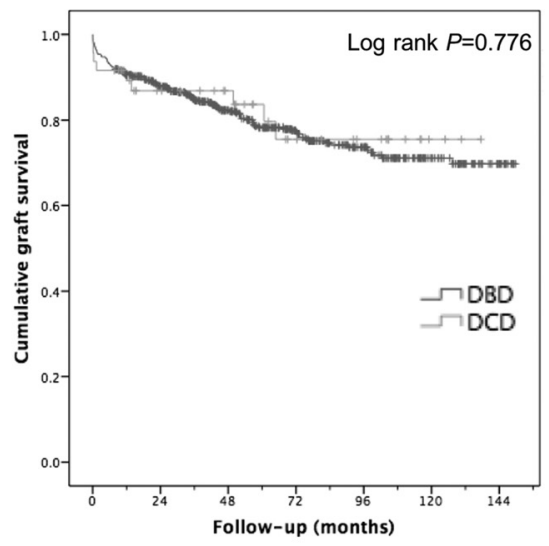

higher donor risk index (Feng et al. 2006) (1.68 vs. 1.56, $P<0.001)$. As per our unit policy, recipients of DCD grafts were less likely to be PSC $(1.9$ vs. 9.0\%, $P=0.076)$ or retransplant patients ( 0 vs. $6 \%, P=0.067$ ). Similarly, DCD recipients did not receive split grafts $(0$ vs. $15.3 \%, P<0.001)$. The proportion of DCDs among utilized grafts increased from $4.8 \%$ (2006-2009) to $7.8 \%$ (2010-2012) and remained stable afterward. 157 patients experienced graft loss during follow-up (31 retransplants, 126 deaths). DCD grafts had similar long term graft survival compared to DBDs, although DCDs recipients with high preLT MELD (>20) appeared to have worse outcomes (figure 1).

Conclusions Long term outcomes of DCD grafts are similar to DBD grafts especially when matched with appropriately selected recipients (first transplant, nonPSC patients with low MELD).

\section{IDDF2019-ABS-0332 COMPARISON OF ANTIBIOTICS FOR THE PRIMARY AND SECONDARY PROPHYLAXIS OF SPONTANEOUS BACTERIAL PERITONITIS: A SYSTEMATIC REVIEW AND NETWORK META-ANALYSIS WITH BAYESIAN APPROACH}

Hariom Soni* ${ }^{*}$ Praveen Kumar, Balaji Bellam, Shubhra Mishra, Dhruv Mahendru, Harshal Mandavdhare, Vishal Sharma. Postgraduate Institute of Medical Education and Research, India

\subsection{6/gutjnl-2019-IDDFabstracts. 15}

Background Spontaneous bacterial peritonitis is an infection of high serum-ascites albumin gradient (SAAG) ascites which usually occurs with cirrhosis. Various antibiotics are used for primary and secondary prevention of spontaneous bacterial peritonitis (SBP). We planned this study to systematically review the evidence from randomised studies comparing various antibiotics with placebo or one another for primary and secondary prevention of SBP.

Methods We did a comprehensive literature search using various databases (i.e. MEDLINE via Ovid and PubMed, Embase, Scopus, Web of science, LILIAC, Cochrane Central Register of Controlled Trials and clinicaltrials.gov from inception to $6^{\text {th }}$ September 2018. The search strategy contained no language restrictions and used following keywords 'Liver cirrhosis', 'Chronic liver disease', 'Chronic Liver Failure', 'Primary

B

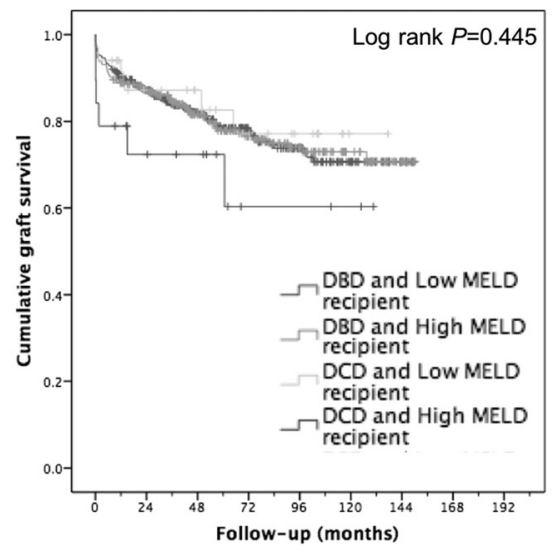


prevention', 'Secondary prevention', Antibiotic, Norfloxacin, Rifaximin, Ciprofloxacin and TMP-SMX. Only those randomised studies were included which evaluated the role of antibiotics in high-risk cirrhosis patients of more than 18 years of age for primary or secondary prophylaxis of spontaneous bacterial peritonitis. Our outcomes were occurrence/recurrence of SBP episode, extra-peritoneal infections, any adverse effects and reduction in mortality. We did random-effects network meta-analysis using a Bayesian approach, and calculated odds ratios (ORs) and 95\% CrI; agents were ranked using rank probabilities.

Results We found total 1211 records in our systematic database search and out of these 17 randomised trials were found eligible for network meta-analysis.

In aggregate group of both primary and secondary SBP, for the outcome of SBP occurrence/recurrence Rifaximin daily, Norfloxacin daily, Rifaximin Norfloxacin daily, Ciprofloxacin intermittent and Trimethoprim-sulfamethoxazole daily were found to be having an odds of $0.050(0.0049-0.21), 0.17$ (0.048-0.41), 0.053(0.0036-0.35), 0.17(0.028-0.76) and 0.16 $(0.027-0.62)$ as compared to that of placebo. The rank probability showed that Rifaximin Norfloxacin daily and Rifaximin daily has a probability of 0.42 and 0.41 respectively for getting ranked as one.

Conclusions CIprofloxacin, Norfloxacin, Trimethoprim-sulfamethoxazole and Rifaximin are useful in prophylaxis of SBP in patients with cirrhosis.

\section{IDDF2019-ABS-0347 PLATELET COUNT AFTER LIVING DONOR LIVER TRANSPLANTATION AS A SURROGATE FOR PORTAL VEIN PRESSURE MONITORING AND PREDICTOR OF GRAFT HYPERPERFUSION SYNDROME: THE '5-67- 8' RULE}

Jeffrey Samuel Co*, CC Wang, CC Yong, CC Lin. Kaohsiung Chang Gung Memorial Hospital, Taiwan

\subsection{6/gutjnl-2019-IDDFabstracts. 16}

Background Thrombocytopenia early after living donor liver transplantation (LDLT) mainly occurs secondary to hepatic/ splenic sequestration, a product of Portal vein pressure (PVP). Portal vein flow (PVF) unlike PVP, is mainly an inflow parameter and does not directly reflect sinusoidal pressure and predict graft dysfunction secondary to hyperperfusion syndrome (HPS). The aim of this study is to determine whether postLDLT platelet count (PC) can reflect PVP and serve as a biomarker to predict HPS.

Methods A total of 757 consecutive adult to adult LDLTs were performed from July 2010 to January 2018 at Kaohsiung Chang Gung Memorial Hospital. After excluding recipients who underwent splenectomy or splenic artery ligation, a total of 690 patients were included. Postoperative liver function, the volume of ascites and graft hemodynamics were recorded on days 1,3,5,7 and 14. Correlation analysis was done using Generalized estimating equations and Receiver operating characteristic analysis (ROC) with Youden's index to determine the optimal cut-off point.

Results A total of 201 patients (29\%) developed HPS in this study. Post LDLT PC significantly correlated to PVF $(p<0.001)$ and HPS $(p<0.001)$. Multiple linear regression analysis showed that PC was a better predictor of HPS when compared to PVF (R2 0.02 vs 0.003 ). PC at postoperative day 5 (POD5) was also a satisfactory biomarker for PVP and predictor of HPS with an AUC of 0.628. Youden's index revealed that the optimal cut-off point for PC was 67,000 per $\mathrm{mm} 3$ with a sensitivity of 0.78 and specificity of 0.59 .

Conclusions Platelet counts within the first 2 weeks after LDLT can serve a surrogate for PVP monitoring and can be used as a guide for further inflow modulation. Furthermore, a PC on POD5 of less than or equal to 67,000 per $\mathrm{mm} 3$ is $80 \%$ sensitive in predicting HPS hence, the ' $5-67$ 8 ' rule.

\section{Basic Gastroenterology}

\begin{tabular}{l|l}
\hline IDDF2019-ABS-0027 & URINARY FORMATE AND GLYCINE ARE \\
& ASSOCIATED WITH TREATMENT RESPONSE \\
& IN PATIENTS TREATED WITH ANTIBIOTICS \\
& FOR POUCHITIS
\end{tabular}

${ }^{1}$ Jonathan Segal ${ }^{*},{ }^{2}$ Magali Sarafian, ${ }^{2}$ Ivan Jose Serrano Contreras, ${ }^{2}$ Alexandros Pechlivanis, ${ }^{2}$ Jerusa Brignardello, ${ }^{2}$ Yih-harn Siaw, ${ }^{2}$ Lucia Braz, ${ }^{2}$ Susan Clark, ${ }^{2}$ Elaine Holmes. ${ }^{1}$ St Mark's Hospital, UK; ${ }^{2}$ Imperial College London, UK

\subsection{6/gutjnl-2019-IDDFabstracts. 17}

Background Restorative proctocolectomy (RPC) is considered the preferred surgical choice for patient with ulcerative colitis (UC) who have failed medical therapy and in some patients with familial adenomatous polyposis (FAP). It has been shown through metabolic profiling of urine that $\mathrm{CD}$ patients have higher levels of formate and lower levels of hippurate and 4-cresol sulfate when compared to healthy controls. To date, extensive metabolic profiling in RPC has yet to be studied.

This study aimed to determine compounds found in urine that are associated with treatment response in patients that have been treated for pouchitis

Methods Patients with pouchitis were recruited from a single centre. Pouchitis was defined using the pouch disease activity index (PDAI) and pouchitis was considered when the score was 37. Response to antibiotics was defined as either a 2 points reduction in PDAI.

Mid-stream morning urine samples were collected. Samples we stored at $-80^{\circ} \mathrm{C}$ until analysis. ${ }^{1} \mathrm{H}$-NMR profile was recorded using the Bruker ${ }^{\circledR}$ Avance III $600 \mathrm{MHz}$ spectrometer, with a Samplejet 96 well autosampler. The full resolution ${ }^{1} \mathrm{H}$ NMR spectra were imported into the SIMCA-P software package, and multivariate data analyses were carried out. Metabolite assignment was performed by comparing chemical shifts, Jres coupling, and peaks multiplicity

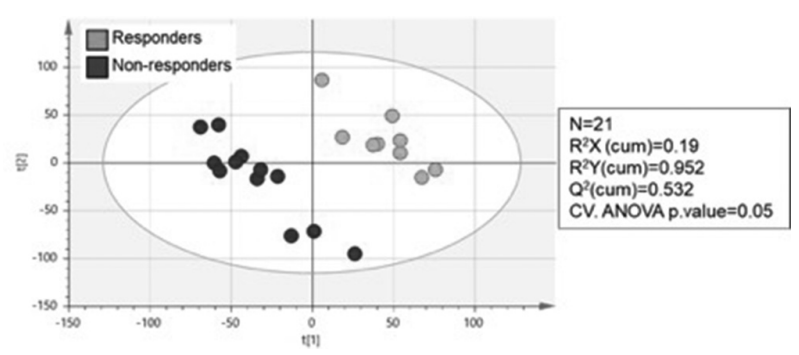

Abstract IDDF2019-ABS-0027 Figure 1 UV PLS DA showing differences between responders vs non-responders 